\title{
Non-visually-guided distance perception depends on matching torso fluctuations between training and test
}

\author{
Dan W. Teng ${ }^{1}$ - Charles L. Eddy ${ }^{1}$ - Damian G. Kelty-Stephen ${ }^{1}$
}

Published online: 14 October 2016

(C) The Psychonomic Society, Inc. 2016

\begin{abstract}
Blindwalking to replicate an instructed distance requires various sensory signals. Recent evidence in movement science across many organisms suggests that multifractal organization of connective tissue supports the use of these signals. Multifractal structure is a multiplicity of power laws defining distribution of proportion across many time scales that helps predict judgments of the objects' length. Present work tests whether the multifractal structure in postural accelerometry during blindwalking predicts blindwalking distance replications. Ten undergraduate student participants each completed 20 trials of distance-perception each comprising two laps. On each Lap 1, experimenters led participants to walk on any of five prescribed distances, randomly assigning half to walk Lap 1 with eyes open and another half to walked Lap 1 with eyes closed. On Lap 2, all participants walked with eyes closed to replicate instructed distances from Lap 1. We collected postural accelerometry from the torso during each lap. Regression modeling showed that multifractality of postural accelerometry on both Lap 1 and Lap 2 contributed significantly to Lap-2 blindwalking responses. According to this model, more accurate Lap-2 replications of Lap-1 distance came from eyes-closed participants whose posture had comparable multifractality on both laps. Multifractality provides insights into the sequence of exploratory behaviors for blindwalking responses to distance perception.
\end{abstract}

Keywords Perception and action $\cdot$ Haptics $\cdot$ Kinesthesis

Damian G. Kelty-Stephen

keltysda@grinnell.edu

1 Psychology Department, Grinnell College, 1115 8th Ave, Grinnell, IA 50112, USA
Distance perception draws on multiple physiological systems. Optical variables inform sighted distance judgments (e.g., Wu, $\mathrm{He}, \&$ Ooi, 2007), and participants with eyes closed can blindly walk a distance commensurate with distance to that target (Thomson, 1983; Elliott, 1986). Blindwalking participants use haptic information from footfalls and postural corrections to feel their way on "how long" it is to the target (Loomis, Da Silva, Fujita, \& Fukusima, 1992; Loomis et al., 1993). Vestibular signals provide self-motion cues (e.g., Etienne \& Jeffery, 2004; McNaughton, Battaglia, Jensen, Moser, \& Moser, 2006). Blindwalking participants must integrate their visual trace with an efference copy encoding muscular effort together with various vestibular, tactile, and proprioceptive signals (Ivanenko, Grasso, Israel, \& Berthoz, 1997).

Signal transmissions from footfall to central executive rely on the nesting of connective tissues across various scales weaving muscle, tendon, bone, and nervous tissue together. Of course, the signals travel through neural tracts in humans, but postural adaptations occur faster than neural transmission allows in multicellular organisms much smaller than humans posing shorter neural distances to traverse (e.g., Endlein \& Federle, 2013) but also in humans, supporting quiet standing (Marsden, Merton, \& Morton, 1983), speech (Kelso, Tuller, Vatikiotis-Bateson, \& Fowler, 1984), and hopping (Moritz \& Farley, 2004). The connective tissues constitute a hierarchically organized network of small prestressed structures (e.g., at the level of actin filaments composing a single cell's skeleton) nested within larger prestressed structures (e.g., extracellular matrix) nested within yet larger pre-stressed structures (e.g., the fascial encasings around muscles and joints). This nested structure allows rapid interactions across multiple scales, supporting rapid anticipatory response to perturbation and making the best use of sometimes sparse stimulation, e.g., allowing us to walk fluidly on feet rather than our hands despite having fewer 


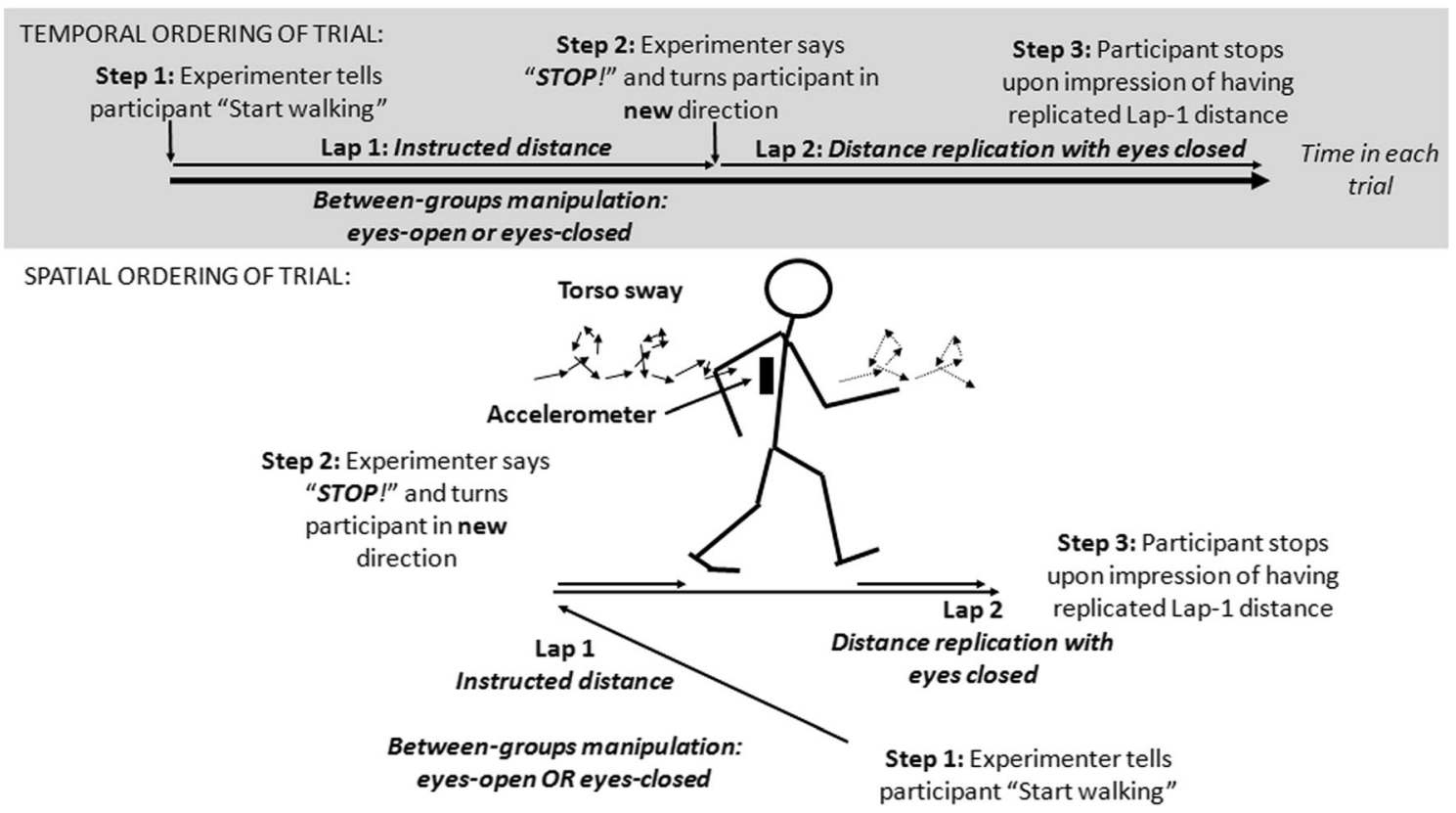

Fig. 1 Panels depicting temporal and spatial organization of a trial (top and bottom panels, respectively)

mechanoreceptors in our feet than in our hands (Turvey \& Fonseca, 2014). For another perceptual example, hair cells in the ear only respond to acoustic stimulation as auditory perception requires because of connective tissues maintaining the tautness of the hair cells (Ingber, 2006). Indeed, this hierarchically nested background support for neural transmission gives human locomotion its unique form (Kiely \& Collins, 2016).

This commitment to hierarchical organization of nested structures interacting across scales entails a specific geometry called a multifractal system (Mandelbrot, 1983). Multifractality refers to a specific case of heterogeneous distribution across measurement scales. Standard linear statistics emphasize the mean and variance of a distribution. However, they assume homogeneity across time, such that the amount of a measured quantity in subsets of the entire sequence should relate to the total amount of a measured quantity in a fixed, single power-law relationship defined by subset size. Sequence subsets comprising half the total-series length will contain .5 proportion of the total-series sum, subsets with one-quarter of the series length contain .25 of the total series sum, etc. Proportion $P$ follows a relationship with subset length $n$, i.e., $P(n) \sim n^{1}$, which trivial relationship only holds for a homogenous series. Multifractal series are those series whose temporal structure is sufficiently heterogeneous across time as to warrant rephrasing the above once-trivial relationship into a more generic $P(n) \sim n^{\alpha}$. Processes built hierarchically allowing interactions across scales exhibit multiples of these power-law relationships between proportion and subset length. These exponents compose the "multifractal spectrum," and its range is the distribution's "multifractality." If the hierarchical relationships across scales of connective tissue provide an important medium for haptic information, then multifractality of blindwalking should contribute to predictions of how well participants estimate path length.

Multifractality is a statistical property definable for a spatial array (e.g., the connective tissues of the human body) as well as for a measurement series (e.g., amount of postural sway over time). Previous work has shown both that multifractality estimated for postural-sway time series successfully predicts how quietly standing participants estimate the lengths of unseen

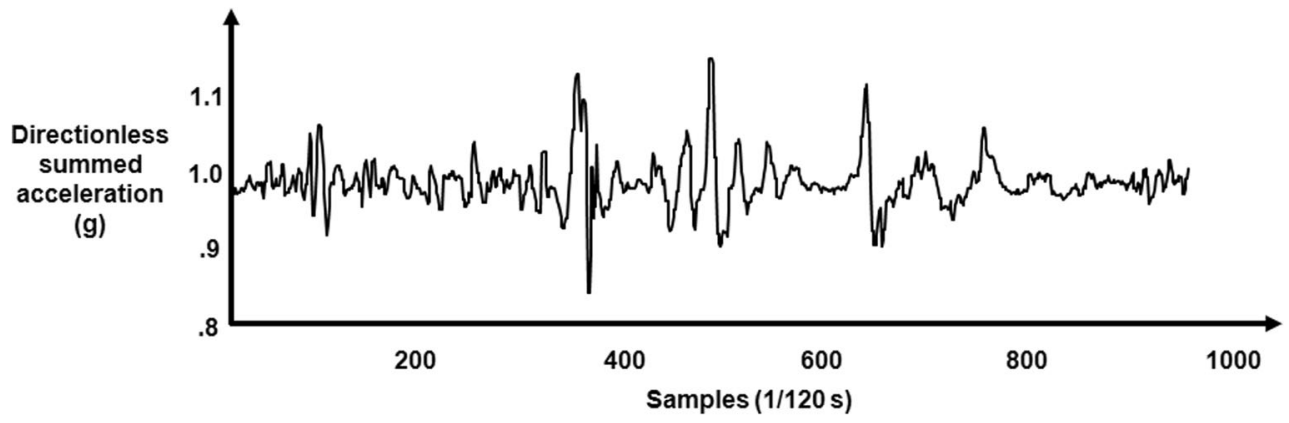

Fig. 2 Example of a postural accelerometry series from a lap of blindwalking. Directionless summed acceleration equals the square root of summed squared accelerations across all axes 


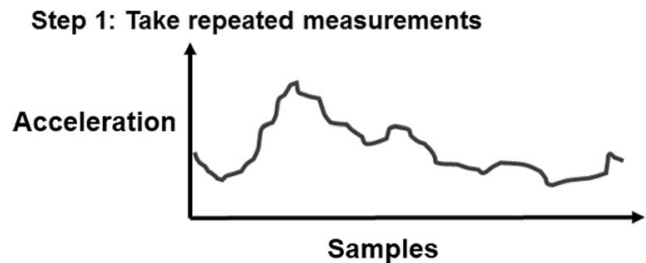

Step 3: Plot average proportion in bins for each $L$

Step 2: Bin series with different bin sizes $L$

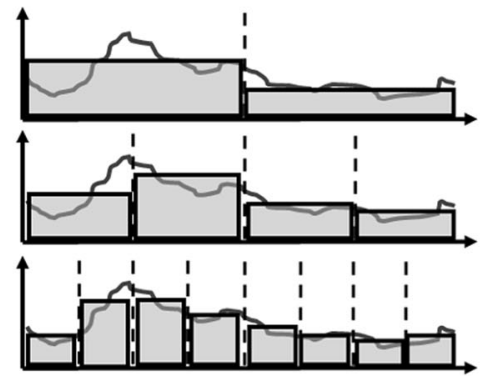

Step 4: Plot Shannon entropy of proportions for each L

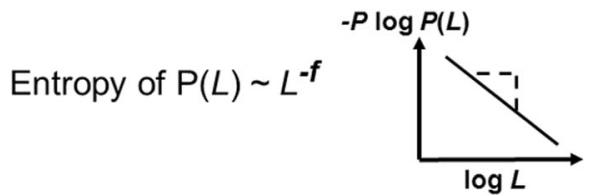

Slope from linear regression yields Hausdorff dimension $f$

Fig. 3 Schematic representation of first steps of a multifractal algorithm that bin a repeated measure on many scales (left panel) and that estimate linear relationships between logarithmic average proportion and bin size as well as between Shannon entropy and bin size

objects felt only haptically against the spine (Palatinus, Dixon, \& Kelty-Stephen, 2013) and that multifractality differs significantly as these same participants learn to judge these objects with selective attention to the whole object or just to one side of it (Palatinus, Kelty-Stephen, Kinsella-Shaw, Carello, \& Turvey, 2014). Touch relies on a similar set of sensory signals to locomotion (Lederman, Klatzky, \& Barber, 1985; Moscatelli, Naceri, \& Ernst, 2014), and, thus, we propose that multifractal temporal structure at the torso during gait may help to predict information collected through locomotion. We should be able to predict changes in the resulting estimation of path length based on changes in the multifractal patterning of torso fluctuations during locomotion.
We recruited participants for a distance-perception task that tested all participants' blindwalking ability. We led them through a conventional procedure (e.g., Sun, Campos, Young, Chan, \& Ellard, 2004), walking distances instructed by the experimenter (Lap 1; L1) and then turning in a different direction to replicate that distance (Lap 2; L2). One group had their eyes closed for the duration of the experiment, and the other group had their eyes open only during L1.

Our reasoning for manipulating vision was that effects of multifractality of the torso should differ when participants had the benefit of visual information. Visual information during walking might diminish the relative importance of haptic information during walking in estimating path length for later

Step 5: Apply " $q$ " exponent to proportions and repeat

Step 6: Plot $(\alpha(q), f(q))$ Steps 3 and 4 for each of many values of $q$

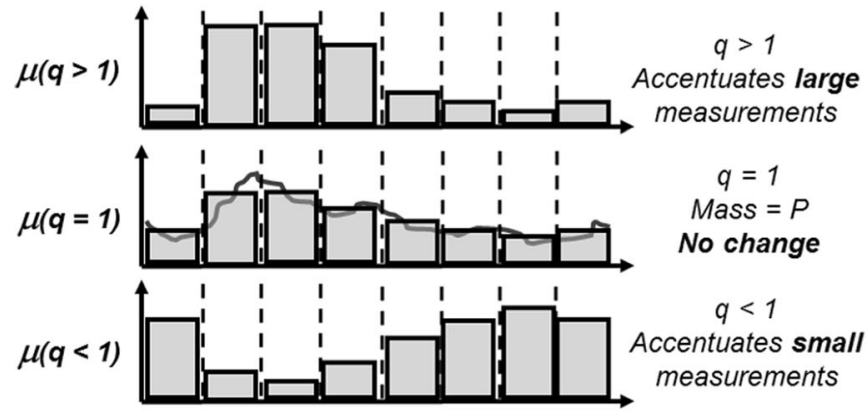

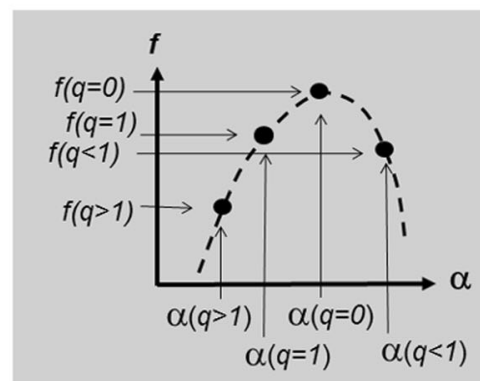

Multifractal Spectrum
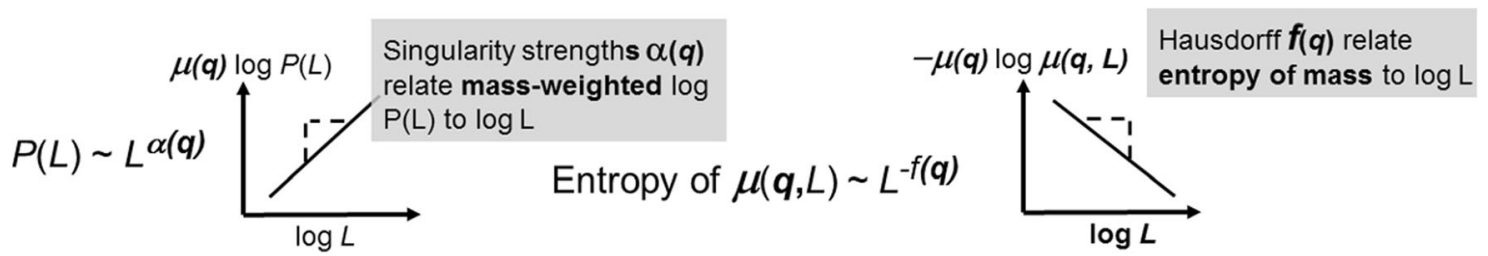

Fig. 4 Concluding steps of multifractal analysis that use parameter $q$ to accentuate proportions of different size (left panel) and then re-estimate the same exponents as in Fig. 3 for each new value of $q$ (bottom panels). The multifractal spectrum comprises ordered pairs of exponents for matching $q$ 
reproduction. Hence, we expected in Hypothesis 1 that effects of $\mathrm{L} 1$ torso multifractality $\left(\mathrm{MF}_{\mathrm{L} 1}\right)$ would depend on the participants having eyes closed on L1. Given the prospective nature of perception (Gibson, 1979), blindwalking might serve not just to maintain upright stance but actually to tune the movement system's multifractal structure towards the intention to later reproduce the distance. Hypothesis 2 was that effects of L2 torso multifractality $\left(\mathrm{MF}_{\mathrm{L} 2}\right)$ on L2 blindwalked distance ( Dist $_{\mathrm{L} 2}$ ) would depend on the participant having their eyes closed for L1. Blindwalking to reproduce a previously seen distance has already shown itself to be an ongoing response that participants make up as they go (Hajnal, Bunch, \& Kelty-Stephen, 2014).

A related question (and Hypothesis 3) was whether multifractality of the torso during L1 or L2 might influence the accuracy of reproduction of Dist $t_{\mathrm{L} 1}$. For instance, the matching of multifractal complexity between L1 and L2 might support a participant's memory for the original distance (L1) when attempting to replicate it (L2). The more similar multifractality is to L1 and L2, the more similarly poised the body's cross-scale interactions should be to support the flow of sensory information during walking. So, we might suppose that the transfer of information gathered during the instructed Lap 1 (L1) would improve recall for instructed distance during Lap 2 (L2), and so perhaps matching multifractality from L1 to L2 strengthens the informational linkage between training and test.

\section{Method}

\section{Participants}

Ten adults (four female, six male) aged 18-22 years participated after providing informed consent according to Grinnell College's Institutional Review Board.

\section{Materials}

Theater-rigging harnesses secured an iPhone running Chris Wozny's "Data Collection" app (https://itunes.apple. com/us/app/data-collection/id479348835? $\mathrm{mt}=8$ ) to the participant's back. The app collected accelerometry at 120 Hz. Surveyor's wheels provided distance measurements.

\section{Design}

Experimenters randomly assigned participants to one of two groups differing only in keeping eyes open or closed during L1.

\section{Procedure}

Experimenters led participants through blindwalking responses on a flat, open grassy field on Grinnell College
Table 1 Coefficients from regression modeling predicting blindwalked distance response (Lap 2) as a function of summed triaxial accelerometry from both Lap 1 and Lap 2 as well as of availability of vision during Lap 1

\begin{tabular}{|c|c|c|c|}
\hline Predictor & $B$ & $S E$ & $p$ \\
\hline Intercept & $4,276.28$ & 843.62 & $<.0001$ \\
\hline \multicolumn{4}{|l|}{ First-lap predictors } \\
\hline Dist $_{\text {Instructed }}$ & .87 & .06 & $<.0001$ \\
\hline Eyes-shut & 356.37 & 127.84 & $<.0001$ \\
\hline Duration $_{\mathrm{L} 1}$ & -.0059 & .0009 & $<.0001$ \\
\hline Mean $_{L 1}$ & 137.59 & 125.67 & .28 \\
\hline $\mathrm{SD}_{\mathrm{L} 1}$ & -66.55 & 38.24 & .08 \\
\hline $\mathrm{MF}_{\mathrm{L} 1}$ & 6.67 & 111.80 & .95 \\
\hline Eyes-shut $\times$ Mean $_{\mathrm{L} 1}$ & -374.44 & 131.36 & $<.01$ \\
\hline Eyes-shut $\times \mathrm{SD}_{\mathrm{L} 1}$ & 101.28 & 35.32 & $<.01$ \\
\hline Eyes-shut $\times \mathrm{MF}_{\mathrm{L} 1}$ & -301.91 & 113.27 & $<.01$ \\
\hline \multicolumn{4}{|l|}{ Second-lap predictors } \\
\hline Duration $_{\mathrm{L} 2}$ & .0071 & .0006 & $<.0001$ \\
\hline Mean $_{\mathrm{L} 2}$ & $-4,414.03$ & 867.49 & $<.0001$ \\
\hline $\mathrm{SD}_{\mathrm{L} 2}$ & $-20,109.82$ & $3,474.38$ & $<.0001$ \\
\hline $\mathrm{MF}_{\mathrm{L} 2}$ & $-68,901.24$ & $17,819.55$ & $<.001$ \\
\hline Eyes-shut $\times \mathrm{MF}_{\mathrm{L} 2}$ & 283.40 & 114.39 & $<.05$ \\
\hline Mean $_{\mathrm{L} 2} \times \mathrm{SD}_{\mathrm{L} 2}$ & $20,161.82$ & $3,483.71$ & $<.0001$ \\
\hline $\operatorname{Mean}_{\mathrm{L} 2} \times \mathrm{MF}_{\mathrm{L} 2}$ & $68,990.40$ & $1,788.63$ & $<.0001$ \\
\hline $\mathrm{SD}_{\mathrm{L} 2} \times \mathrm{MF}_{\mathrm{L} 2}$ & $332,138.71$ & $7,291.04$ & $<.0001$ \\
\hline $\operatorname{Mean}_{\mathrm{L} 2} \times \mathrm{SD}_{\mathrm{L} 2} \times \mathrm{MF}_{\mathrm{L} 2}$ & $-322,316.42$ & $7,298.19$ & $<.0001$ \\
\hline
\end{tabular}

campus for five instructed distances $(10 \mathrm{~m}, 20 \mathrm{~m}, 30 \mathrm{~m}$, $40 \mathrm{~m}$, and $50 \mathrm{~m}$ ), each presented once in each of four blocks randomized within blocks (i.e., a total of 20 trials). Participants walked at a self-selected comfortable speed. Experimenters explicitly instructed participants not to count steps and further prevented step-counting by engaging participants in small talk.

Each trial comprised two laps (Fig. 1). Lap 1 (L1) involved experimenters starting accelerometry, instructing participants when to start and stop walking. Participants with eyes open for L1 closed their eyes and experimenters turned participants in a randomly selected direction, and Lap 2 (L2) involved participants blindwalking forward

Table 2 Coefficients from dramatically smaller regression model predicting blindwalked distance response (Lap 2) as a function of summed triaxial accelerometry from both Lap 1 and Lap 2

\begin{tabular}{lrrl}
\hline Predictor & \multicolumn{1}{l}{$B$} & \multicolumn{1}{l}{$S E$} & $p$ \\
\hline Intercept & 1.34 & 4.28 & $<.0001$ \\
Dist $_{\text {Instructed }}$ & .97 & .05 & $<.0001$ \\
Eyes-shut & 3.43 & 1.39 & $<.0001$ \\
$\mathrm{MF}_{\mathrm{L} 1}$ & 305.77 & 78.64 & $<.0001$ \\
$\mathrm{MF}_{\mathrm{L} 2}$ & -324.39 & 72.11 & $<.001$ \\
\hline
\end{tabular}




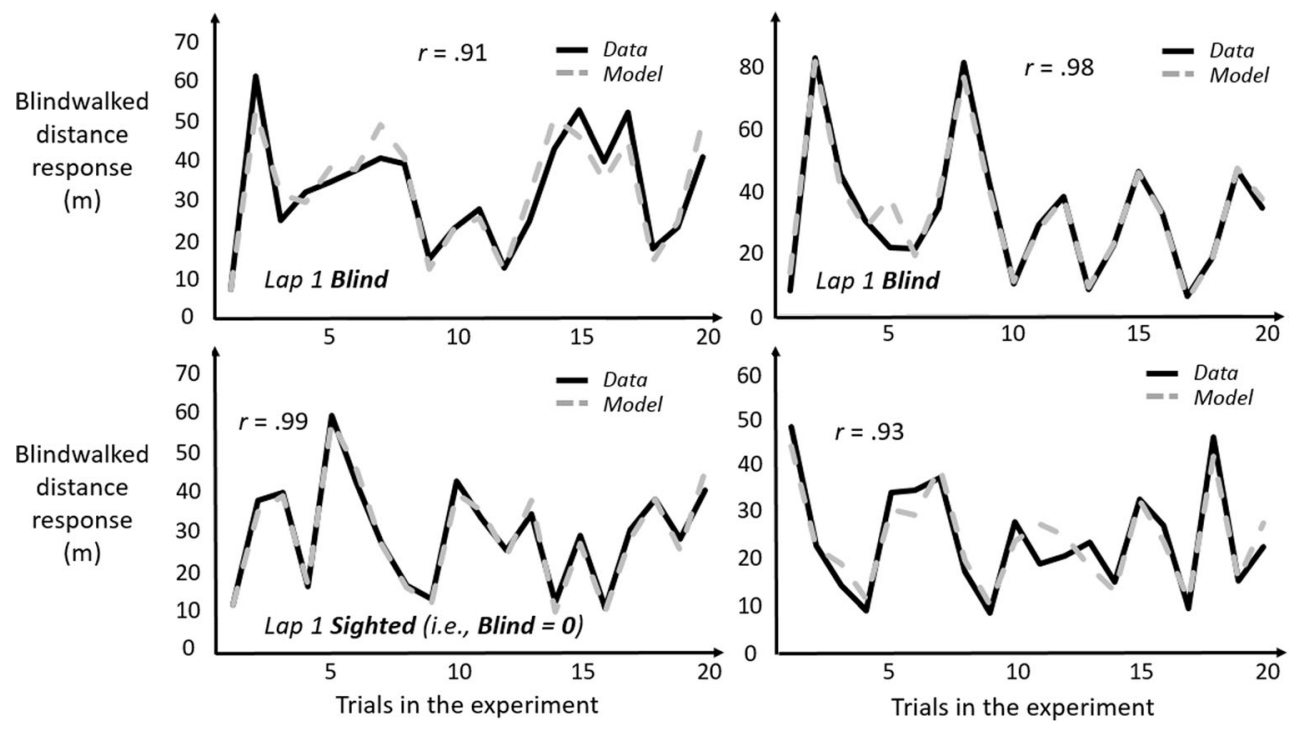

Fig. 5 Example of Lap-2 blindwalking responses (black solid lines) with regression-model predictions on each trial (gray dashed lines) from both groups and for participants with strongest and weakest agreement according to correlation coefficient $r$

until they felt that they had replicated the instructed distance. Experimenters followed participants to prevent collisions, but left them free to choose when to stop, stopping accelerometry after the participant stopped. Despite always walking forward, participants did not walk in completely straight paths. Experimenters measured total path length; instruction was to replicate distance and not to locate a starting point (e.g., Harrison \& Turvey, 2010).

\section{Data analysis}

Experimenters recorded triaxial accelerometry for each lap. The square root of the sum of squared acceleration in each of three axes provided an unsigned accelerometry series (Fig. 2).

Table 3 Lap-2 blindwalking responses for participants randomly assigned to the condition with eyes open for Lap 1

\begin{tabular}{|c|c|c|c|c|}
\hline \multirow[t]{2}{*}{$\begin{array}{l}\text { Instructed distance } \\
\text { (Dist } \\
\text { Intended })\end{array}$} & \multicolumn{2}{|c|}{$\begin{array}{l}\text { High Lap-1 } \\
\text { multifractality }\left(\mathrm{MF}_{\mathrm{L} 1}\right)\end{array}$} & \multicolumn{2}{|c|}{$\begin{array}{l}\text { Low Lap-1 } \\
\text { multifractality }\left(\mathrm{MF}_{\mathrm{L} 1}\right)\end{array}$} \\
\hline & Low $\mathrm{MF}_{\mathrm{L} 2}$ & High $\mathrm{MF}_{\mathrm{L} 2}$ & Low $\mathrm{MF}_{\mathrm{L} 2}$ & High $\mathrm{MF}_{\mathrm{L} 2}$ \\
\hline 10 & 11.17 & 11.71 & 11.07 & 11.62 \\
\hline 20 & 19.86 & 20.40 & 19.76 & 20.31 \\
\hline 30 & 28.55 & 29.09 & 28.45 & 29.00 \\
\hline 40 & 37.24 & 37.78 & 37.14 & 37.69 \\
\hline 50 & 45.93 & 46.47 & 45.83 & 46.38 \\
\hline
\end{tabular}

Note: $\mathrm{MF}_{\mathrm{L} 1}$ and $\mathrm{MF}_{\mathrm{L} 2}$ are both scalar values estimated on a continuous positive scale and we only use the third and first quartiles to define the "high" and "low" values of these variables for the purposes of depicting what the model in Table 1 predicts for a hypothetical participant with relatively high and relatively low multifractal-spectrum width $\mathrm{MF}_{\mathrm{L} 1}$ or $\mathrm{MF}_{\mathrm{L} 2}$
Multifractal algorithm The quasiperiodic accelerometry warranted the proportion-based multifractal analysis (Chhabra \& Jensen, 1989). Multifractal analysis diagnoses homogeneity or heterogeneity of repeated measures. It partitions (or "bins") the series according to various bin sizes, usually from four points to a fourth of series length (Fig. 3; left panel).

Multifractal analysis continues by calculating statistics on binned quantities and examining how these statistics change with bin size, i.e., time scale. Next, it estimates exponent $\alpha$ defining how average bin proportion grows with bin size and an $f$ exponent relating bin size to Shannon entropy (Shannon, 1948).

The procedure repeats for different settings of a parameter $q$. Proportions raised to exponent $q$ provides "mass" $\mu$ to weight proportions selectively according to proportion size (see Fig. 4, left panel). $q$-Based variability with mass $\mu(q)$ generalizes single $\alpha$ and $f$ estimates from earlier steps into continua $\alpha(q)$ and $f(q)$. Multifractal analysis distinguishes temporally heterogeneous series from homogenous series by the amount of variety in $\alpha(q)$ and $f(q)$. Plotting $\alpha(q)$ and $f(q)$ as ordered pairs yields the multifractal spectrum, an often-asymmetric and inverted U-shaped curve (Fig. 4, right panel). We included $\alpha(q)$ and $f(q)$ whenever log-scaled linear fits correlated at $r$ of .995 for the log-scaled linear fit. We excluded values of $q$ either (1) for which mass-weighted proportion and for which Shannon entropy were undefined (e.g., due to masses rounding to zero) or (2) power-law fits for which $r<.995$. $^{1}$

\footnotetext{
${ }^{1}$ See the Appendix for algebraic representation of the algorithm as well as a brief step-by-step example using an extremely small 32-measurement series that outlines the calculation of each bin proportion and mass, borrowing from a tutorial on multifractal analysis (Kelty-Stephen, Palatinus, Saltzman, \& Dixon, 2013). Link: http://sites.google.com/site/foovian/PPSR-16-021Supp.pdf.
} 


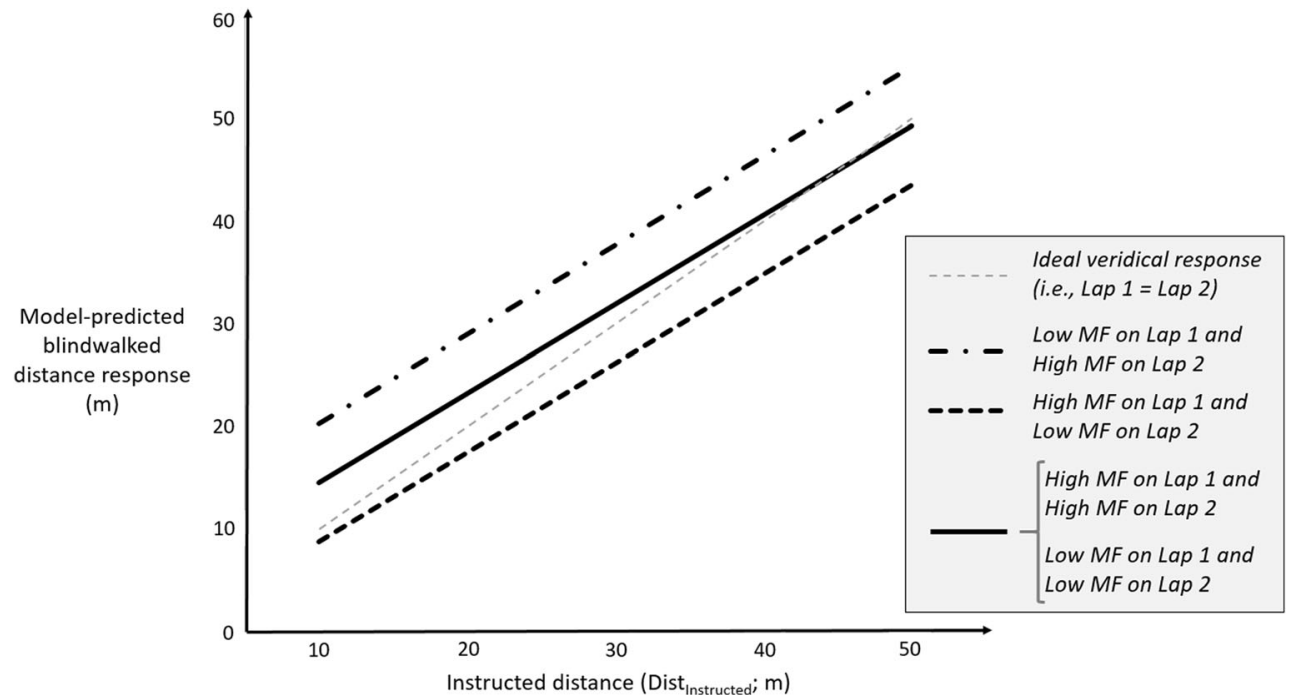

Fig. 6 Model-predicted Lap-2 blindwalked responses as a function of Lap-1 instructed distance and of multifractal-spectrum width for participants with eyes closed compared to veridical responses (gray dashed line). Third and first quartiles serve as "high" and "low"

Subsequent modeling focused on the dependent variable of Dist $_{\mathrm{L} 2}$. We used function "lmer" in the R package "lme4" for linear mixed-effect modeling (Bates, Maechler, Bolker, \& Walker, 2015) as well as R package "ImerTest" for the Satterthwaite estimation of $F$-test-based $p$-values for fixed effects (Kuznetzova, Brockhoff, \& Christensen, 2016). We estimated coefficients for the predictors of instructed distance (Dist Instructed $)$, eyes' openness on Lap 1 (Eyes-shut $=1$ for closed, 0 for open), as well as all of the following numeric values: duration of each lap in terms of $120-\mathrm{Hz}$ accelerometry samples Duration $_{\mathrm{L} 1}$ and Duration ${ }_{\mathrm{L} 2}$ for $\mathrm{L} 1$ and L2, respectively), mean acceleration $\left(\mathrm{Mean}_{\mathrm{L} 1}\right.$ and $\left.\mathrm{Mean}_{\mathrm{L} 2}\right)$, standard deviation of acceleration $\left(\mathrm{SD}_{\mathrm{L} 1}\right.$ and $\left.\mathrm{SD}_{\mathrm{L} 2}\right)$, and multifractalspectrum width $\left(\mathrm{MF}_{\mathrm{L} 1}\right.$ and $\left.\mathrm{MF}_{\mathrm{L} 2}\right)$. We fitted only a randomeffect intercept for individual participants. The fixed-effects equation was as follows:

$$
\begin{aligned}
\text { Dist }_{\text {Instructed }} & + \text { Eyesshut }+ \text { Duration }_{L 1}+\text { Duration }_{L 2}+\text { Eyesshut }^{*} \text { Mean }_{L 1} \\
& + \text { Eyesshut }^{*} D_{L 1}+\text { Eyesshut }^{*} M F_{L 1}+\text { Eyesshut }^{*} M F_{L 2} \\
& + \text { Mean }_{L 2} * S D_{L 2} * M F_{L 2}
\end{aligned}
$$

as well as all lower-order interactions and main effects included in the foregoing terms.

\section{Results and discussion}

Dist $_{\mathrm{L} 2}$ correlated with Dist $\mathrm{L}_{1}, r=.84$. Also, summed triaxial accelerometry exhibited extremely similar descriptive statistics between L1 and L2 with mean time-series durations $3,335.35$ and 3,354.61 1/120-s frames $(S E \mathrm{~s}=111.18$ and 129.92), average triaxial acceleration per sample 1.0029 and 1.0021 gravities $(S E \mathrm{~s}$ both $=.0007)$, and standard deviation of multifractal (MF) values to portray Table 1 model predictions for hypothetical participants with high and low multifractal-spectrum width $\mathrm{MF}_{\mathrm{L} 1}$ or $\mathrm{MF}_{\mathrm{L} 2}$

triaxial acceleration per 120-Hz sample SDs .23 and .23 (SEs $=.0031$ and .0032 ), respectively.

All predictors were significant except for Lap-1 main effects of Mean, SD, and MF (Table 1). ${ }^{2}$ The model reiterates the strong positive relationship between instructed distance and $\operatorname{Dist}_{\mathrm{L} 2}(B=$ $.87, S E=.06, p<.0001)$. Dist ${ }_{\mathrm{L} 2}$ covaried with duration of L2 accelerometry (i.e., the time spent walking, Duration ${ }_{\mathrm{L} 2}, B=$ $.0071, S E=.0006, p<.0001)$, but varied inversely with duration of L1 accelerometry (Duration ${ }_{\mathrm{L} 1}, B=-.0059, S E=.0009, p<$ $.0001)$. A dramatically reduced model including only Dist $_{\text {Instructed }}$, Eyes-shut, $\mathrm{MF}_{\mathrm{L} 1}$, and $\mathrm{MF}_{\mathrm{L} 2}$ showed significant effects of both laps' multifractality for all participants with effects in the same directions (Table 2) as in Table 1. Given that effects for multifractal spectrum width might be confounded with effects moving more or moving more variably (i.e., mean or variance; Kantelhardt et al., 2002), the larger model will be more conservative for interpretation. Because interactions between Eyes-shut and $\mathrm{MF}_{\mathrm{L} 1}$ or $\mathrm{MF}_{\mathrm{L} 2}$ fail to improve the reduced model, this reduced model leaves us with the strange entailment that torso multifractality of both laps contributes significantly for all participants, regardless of whether they have the benefit of visual input. In any event, the larger model is an overwhelming improvement upon the reduced model, $\chi^{2}(12)=167.76$, $p<.0001$.

Regression-model predictions correlated with trial-by-trial Dist $_{\mathrm{L} 2}$ responses, $r=.96$ (Fig. 5).

\footnotetext{
${ }^{2}$ We maintain the heuristic 10-to-1 observation-to-predictor ratio to prevent overfitting (e.g., Babylak, 2004; Finlay, 2014; Harrell, Lee, \& Mark, 1996). Resampling L2 data with replacement 1,000 times showed adequate power for all predictors but $\mathrm{MF}_{\mathrm{L} 1}$, exhibiting nonzero coefficients for $>80 \%$ of the 1,000 resamplings (e.g., Yuan \& Hayashi, 2003).
} 
Hypothesis 1: Lap-1 postural accelerometry contributes to Lap-2 responses for participants with eyes closed Closed eyes during L1 led participants to overestimate Dist $_{\mathrm{L} 2}$ (Eyes-shut, $B=356.37, S E=127.84, p<.0001$ ) but also exhibited significant L1 accelerometry effects on Lap-2 blindwalking distance responses for $\mathrm{Mean}_{\mathrm{L} 1}$, $\mathrm{SD}_{\mathrm{L} 1}$, and $\mathrm{MF}_{\mathrm{L} 1}(B \mathrm{~s}=-374.44,101.28$, and -301.91, $S E \mathrm{~s}=131.36,35.32$, and 113.27 , respectively, $p \mathrm{~s}$ all < $.01)$ on Dist $_{\mathrm{L} 2}$.

Hypothesis 2: Lap-2 multifractality contributes to Lap-2 responses for participants with eyes closed in Lap 1 Main effects and full-factorial interactions of $\mathrm{Mean}_{\mathrm{L} 2}, \mathrm{SD}_{\mathrm{L} 2}$, and $\mathrm{MF}_{\mathrm{L} 2}$ were significant $(p \mathrm{~s}<.001)$, with $\mathrm{Mean}_{\mathrm{L} 2}$ and $\mathrm{SD}_{\mathrm{L} 2}$ moderating effects of $\mathrm{MF}_{\mathrm{L} 2}$ such that, for average values of Mean $_{\mathrm{L} 2}$ and $\mathrm{SD}_{\mathrm{L} 2}$, they reduce predicted effects of $\mathrm{MF}_{\mathrm{L} 2}$ to near zero. However, the positive effect of Eyes-shut $\times \mathrm{MF}_{\mathrm{L} 2}(B$ $=283.40, S E=114.39, p<.05)$ indicates that having experienced L1 with eyes closed amplifies a positive effect of $\mathrm{MF}_{\mathrm{L} 2}$ predicting greater Dist $_{\mathrm{L} 2}$.

Hypothesis 3: Participants whose Lap-2 multifractality matches Lap-1 multifractality have the most accurate Lap-2 responses $M F_{L 1}$ and $M F_{L 2}$ showed no effect on Dist $_{\mathrm{L} 2}$ for participants with eyes open during L1 (Table 3). However, multifractal-spectrum width had stronger effects on the veridicality of Dist $_{\mathrm{L} 2}$ for those participants experiencing L1 with eyes closed. Figure 6 plots regression-modeling predictions for different combinations of low and high multifractal-spectrum widths across L1 and L2. Figure 6 contains a grey dashed line indicating the ideally veridical Lap-2 responses. Regression modeling predicted more accurate L2 responses for participants with comparable multifractal-spectrum width on both laps, i.e., high $\mathrm{MF}_{\mathrm{L} 1}$ and high $\mathrm{MF}_{\mathrm{L} 2}$, or low $\mathrm{MF}_{\mathrm{L} 1}$ and then low $\mathrm{MF}_{\mathrm{L} 2}$. Regression-modeling predictions for these low-matching or high-matching participants differed slightly, but the predicted values were so close that the lines for each on Fig. 6 are visibly indistinguishable.

Under- and over-estimation of Dist $_{\mathrm{L} 2}$ under disparate pairings of different multifractal-spectrum widths warrants further interpretation. Low and high multifractality indicates heterogeneity reflecting sparsity and rich diversity, respectively. Thus, when higher $\mathrm{MF}_{\mathrm{L} 2}$ follows lower $\mathrm{MF}_{\mathrm{L} 1}$, it may indicate a distance-replication walk whose exploratory capacity draws a far richer amount of information, opening the floodgates for stimulus energy and allowing extraneous information potentially distracting to path-length estimation. Conversely, lower $\mathrm{MF}_{\mathrm{L} 2}$ following higher $\mathrm{MF}_{\mathrm{L} 1}$ might indicate a replication blindwalk too constrictive to draw in the requisite amount of information for a path length commensurate to the first lap.
The present study offers an early multifractal view of exploratory properties of postural accelerations in blindwalking. First, these findings may account for effort effects on blindwalking responses previously quantified as average walking speed (Hajnal, Bunch, \& Kelty-Stephen, 2014). Second, the benefit of multifractal fluctuations to touch during quiet standing appears to extend to distance perception by blindwalking. Finally, the matching of multifractal fluctuations may support coordination for haptic information from training (e.g., Lap 1) to test (e.g., Lap 2). When human participants attempt to coordinate with unpredictable stimulus, they will unwittingly exhibit similar multifractal structure to the unpredictable stimulus (Stephen \& Dixon, 2011). Conversely, similar complexity can facilitate communication between two separate systems (West, Geneston, \& Grigolini, 2008). Similar multifractality may facilitate memory-like communication between past and present states of the same organism. More generically, multifractality is an important window on the sequence implicit in exploratory behavior, quantifying the trajectory from curious, uncertain exploration to satisfaction with a complete response.

Acknowledgments D.W.T., C.L.E., and D.G.K.-S. would like to acknowledge the generous support of Grinnell College's Mentored Advanced Project program.

\section{Appendix}

Chhabra and Jensen's (1989) multifractal analysis for nonnegative length-N series $x(t)$

Step 1: Bin $x(t)$ using $N_{n}$ nonoverlapping windows of length $4 \leq n \leq N / 4$, where $N_{n}$ is the greatest integer $\leq N / n$.

Step 2: For each $n$, calculate proportion $P$ for $i$ th bin $\left(i=1,2, \ldots, N_{n}-1, N_{n}\right)$,

$P_{i}(n)=\frac{\sum_{k=n(i-1)+1}^{n i} x(k)}{\sum_{m=1}^{n N_{n}} x(m)}$,

Multifractal analysis examines proportion growth with $n$. For homogeneous series, $P_{i}(n)$ approximately follows one power law:

$$
\begin{gathered}
P(n) \sim n^{1} \\
P\left(N_{n}\right) \sim N_{n}^{-1} .
\end{gathered}
$$

Otherwise, $P_{i}(n)$ may vary across bins, yielding

$$
\begin{gathered}
P(n) \sim n^{\alpha(q)}, \\
P\left(N_{n}\right) \sim N_{n}^{-\alpha(q)} .
\end{gathered}
$$


with real values for $\alpha(q)$ estimated by weighting $P_{i}(n)$ with "mass" (Step 3).

Step 3: Calculate mass $\mu_{i}(q, n)$ :

$$
\mu_{i}(q, n)=\frac{\left[P_{i}(n)\right]^{q}}{\sum_{j=1}^{N_{n}}\left[P_{i}(n)\right]^{q}}
$$

emphasizing higher or lower proportions for $q>1$ or $q<1$, respectively.

Step 4: Calculate $\alpha(q)$ as

$$
\begin{array}{r}
\alpha(q)=\frac{\sum_{i=1}^{N_{n}} \mu_{i}(q) \log P_{i}(n)}{\log n}, \\
\alpha(q)=-\frac{\sum_{i=1}^{N_{n}} \mu_{i}(q) \log P_{i}\left(N_{n}\right)}{\log N_{n}},
\end{array}
$$

when numerator and denominator correlate, $r \geq 995$. If Shannon entropy of $\mu_{i}(q, n)$ yields a linear relationship with $\log n$, then

$$
\begin{array}{r}
f(q)=\frac{\sum_{i=1}^{N_{n}} \mu_{i}(q, n) \log \mu_{i}(q, n)}{\log n} \\
f(q)=-\frac{\sum_{i=1}^{N_{n}} \mu_{i}(q, n) \log \mu_{i}(q, n)}{\log N_{n}}
\end{array}
$$

for a single point $f(\alpha(q))$ in the multifractal spectrum.

Step 5: Recalculate $f(q)$ and $\alpha(q)$ allowing relationships in Eqs. A5 and A6 to drop below $r=.995$ at both extrema for $q$. We used range $-300 \leq q \leq 300$, incremented by .5 .

Step 6: Spectrum width is $\max (\alpha(q))-\min (\alpha(q))$ where $f(\mathrm{q})$ is defined.

\section{References}

Babylak, M. A. (2004). What you see may not be what you get: A brief, nontechnical introduction to overfitting in regression-type models. Psychosomatic Medicine, 66, 411-421.

Bates, D., Maechler, M., Bolker, B., \& Walker, S. (2015). Fitting linear mixed-effects models using lme4. Journal of Statistical Software, 67, 1-48.

Chhabra, A., \& Jensen, R. V. (1989). Direct determination of the $f(\alpha)$ singularity spectrum. Physical Review Letters, 62, 1327-1330.

Elliott, D. (1986). Continuous visual information may be important after all: A failure to replicate Thomson (1983). Journal of Experimental Psychology: Human Perception \& Performance, 12, 388-391.

Endlein, T., \& Federle, W. (2013). Rapid preflexes in smooth adhesive pads of insects prevent sudden detachment. Proceedings of the Royal Society B, 280, 20122868.
Etienne, A. S., \& Jeffery, K. J. (2004). Path integration in mammals. Hippocampus, 14, 180-192.

Finlay, S. (2014). Predictive analytics, data mining, and big data: Myths, misconceptions and methods. New York: Palgrave Macmillan.

Gibson, J. J. (1979). The ecological approach to visual perception. Boston: Houghton Mifflin.

Hajnal, A., Bunch, D., \& Kelty-Stephen, D. G. (2014). Going for distance and going for speed: Effort and optical variables shape information for distance perception from observation to response. Attention, Perception, \& Psychophysics, 76, 1015-1035.

Harrell, F. E., Lee, K. L., \& Mark, D. B. (1996). Tutorial in biostatistics: Multivariable prognostic models: Issues in developing models, evaluating assumptions and adequacy, and measuring and reducing errors. Statistics in Medicine, 15, 361-387.

Harrison, S. J., \& Turvey, M. T. (2010). Place learning by mechanical contact. Journal of Experimental Biology, 213, 1436-1442.

Ingber, D. E. (2006). Cellular mechanotransduction: Putting all the pieces together again. FASEB Journal, 20, 811-827.

Ivanenko, Y. P., Grasso, R., Israel, I., \& Berthoz, A. (1997). The contribution of otoliths and semicircular canals to the perception of twodimensional passive whole-body motion in humans. Journal of Physiology, 502, 223-233.

Kantelhardt, J. W., Zschiegner, S. A., Koscielny-Bunde, E., Havlin, S., Bunde, A., \& Stanley, H. E. (2002). Multifractal detrended fluctaution analysis of nonstationary time series. Physica A, $316,87-114$.

Kelso, J. A. S., Tuller, B., Vatikiotis-Bateson, E., \& Fowler, C. A. (1984), Functionally specific articulatory cooperation following jaw perturbations during speech: Evidence for coordinative structures. Journal of Experimental Psychology: Human Perception \& Performance, 10, 812-813.

Kelty-Stephen, D. G., Palatinus, K., Saltzman, E., \& Dixon, J. A. (2013). A tutorial on multifractality, cascades, and interactivity for empirical time series in ecological science. Ecological Psychology, 25, 1-62.

Kiely, J., \& Collins, D. J. (2016). Uniqueness of human running coordination: The integration of modern and ancient evolutionary innovations. Frontiers in Psychology, 7, 262.

Kuznetzova, A., Brockhoff, P. B., \& Christensen, R. H. B. (2016). lmerTest: Tests in linear mixed-effect models. $\mathrm{R}$ package version 2.0-30. https://CRAN.R-project.org/package=lmerTest

Lederman, S. J., Klatzky, R. L., \& Barber, P. O. (1985). Spatial and movement-based heuristics for encoding pattern information through touch. Journal of experimental psychology: General, 114(1), 33-49.

Loomis, J. M., Da Silva, J. A., Fujita, N., \& Fukusima, S. S. (1992). Visual space perception and visually directed action. Journal of Experimental Psychology: Human Perception and Performance, $18,906-921$

Loomis, J. M., Klatzky, R. L., Golledge, R. G., Cicinelli, J. G., Pellegrino, J. W., \& Fry, P. A. (1993). Nonvisual navigation by blind and sighted: Assessment of path integration ability. Journal of Experimental Psychology: General, 122(1), 73-91.

Mandelbrot, B. B. (1983). The fractal geometry of nature. San Francisco, CA: Freeman.

Marsden, C. D., Merton, P. A., \& Morton, H. B. (1983). Rapid postural reactions to mechanical displacement of the hand in man. Advances in Neurology, 39, 645-659.

McNaughton, B. L., Battaglia, F. P., Jensen, O., Moser, E. I., \& Moser, M. B. (2006). Path integration and the neural basis of the "cognitive map.". Nature Reviews Neuroscience, 7, 663-678.

Moritz, C. T., \& Farley, C. T. (2004). Human hopping on very soft elastic surface: Implications for muscle pre-stretch and elastic energy storage in locomotion. Journal of Experimental Biology, 208, 939-949.

Moscatelli, A., Naceri, A., \& Ernst, M. O. (2014). Path integration in tactile perception of shapes. Behavioural Brain Research, 274, 355-364. 
Palatinus, Z., Dixon, J. A., \& Kelty-Stephen, D. G. (2013). Fractal fluctuations in quiet standing predict the use of mechanical information for haptic perception. Annals of Biomedical Engineering, 41, 1625-1634.

Palatinus, Z., Kelty-Stephen, D. G., Kinsella-Shaw, J. M., Carello, C., \& Turvey, M. T. (2014). Haptic perceptual intent in quiet standing affects multifractal scaling of postural fluctuations. Journal of Experimental Psychology: Human Perception \& Performance, 40, 1808-1818.

Shannon, C. (1948). A mathematical theory of communication. Bell System Technical Journal, 27, 379-423.

Stephen, D. G., \& Dixon, J. A. (2011). Strong anticipation: Multifractal cascade dynamics modulate scaling in synchronizing behaviors. Chaos, Solitons \& Fractals, 44, 160-168.

Sun, H.-J., Campos, J. L., Young, M., Chan, G. S. W., \& Ellard, C. G. (2004). The contributions of static visual cues, nonvisual cues, and optic flow in distance estimation. Perception, 33, 49-65.
Thomson, J. A. (1983). Is continuous visual monitoring necessary in visually guided locomotion. Journal of Experimental Psychology: Human Perception \& Performance, 9, 427-433.

Turvey, M. T., \& Fonseca, S. T. (2014). The medium of haptic perception: A tensegrity hypothesis. Journal of Motor Behavior, 46, 143-187.

West, B. J., Geneston, E. L., \& Grigolini, P. (2008). Maximizing information exchange between complex networks. Physics Reports, 468, 1-99.

Wu, B., He, Z. J., \& Ooi, T. L. (2007). Inaccurate representation of the ground surface beyond a texture boundary. Perception, 36, 703-721.

Yuan, K. H., \& Hayashi, K. (2003). Bootstrap approach to inference and power analysis based on three test statistics for covariance structure models. British Journal of Mathematical and Statistical Psychology, 56, 93-110. 